\title{
Programas y servicios para personas mayores en centros de fitness de Sevilla Programs and services for older people in fitness centers in Seville
}

\author{
*Jerónimo García Fernández, Jesús Fernández Gavira \\ Universidad de Sevilla(España)
}

\begin{abstract}
Resumen: La práctica de actividad física por parte de las personas mayores, es cada vez más una práctica habitual en este grupo de población. Así las empresas de servicios deportivos, ofertan programas espeć́ficos y desarrollan actividades con el fin de mejorar la calidad de vida de esta población. Uno de los lugares donde se ofertan estas actividades, son los centros de fitness privados (CFP), y cada vez más, se pueden ver usuarios mayores realizando ejercicios en maquinaria de última generación, y realizando actividades con material sofisticado. Nuestro estudio refleja la realidad de 45 CFP del municipio de Sevilla, en relación a las cuotas y actividades específicas ofertadas para los mayores en los propios centros. Del mismo modo, pone de manifiesto la situación de esta oferta en relación a los distritos censales de Sevilla. En la investigación se pudo comprobar, como la mayoría de los centros testados no tenían una oferta específica de actividades para mayores.
\end{abstract}

Palabra clave: Centros de Fitness, Personas Mayores, Cuotas, Servicios, Actividades.

\begin{abstract}
The practice of physical activity by older people is increasingly becoming very usual within this population. Therefore, the sports facilities, are offering specific programs and activities to improve the quality of life for this population. One of the places where these activities are offered, are the private fitness centers (PFC), where nowadays is getting more frequent to find seniors doing exercises in the latest generation apparatus and dealing with modern equipment.Our study reflects the reality of 45 PFC of a Spanish city, Seville, in relation to specific fees and activities offered for seniors at these centers. Similarly, it shows the status of this offer in relation to the census districts of Seville. The research found out that most of the centers analyzed did not have a specific program with activities for seniors.
\end{abstract}

Key words: Fitness Centers, Seniors, Fees, Services, Activities.

\section{Introducción}

La tendencia de la sociedad actual, es que cada vez más exista mayor población mayor, con la repercusión económica, social y política que esto desencadena. A su vez, este aumento de la población mayor, está haciendo que prolifere una industria de productos y servicios dedicados exclusivamente a ellos.

El deporte, como producto emergente en nuestra sociedad, también se adapta a este nuevo grupo de población, y crea y gestiona servicios con el objetivo de paliar las necesidades que demandan. Por ello, es necesario realizar un cambio en la gestión de los servicios, recopilando nuevos y especializados recursos humanos, material y equipamiento, $\mathrm{y}$ con una infraestructura adecuada a la demanda existente.

Es en este punto, donde nos vamos a centrar, más concretamente en las cuotas específicas y actividades dirigidas específicas que se ofertan para los mayores en centros de fitness privados (CFP). Además, dividimos esta oferta según los distritos censales del municipio de Sevilla, haciendo una comparación con la población mayor, y el número de entidades encuestadas en cada distrito.

La investigación en este tipo de centros está siendo orientada hacia la calidad del servicio, satisfacción del usuario y fidelización de los mismos (Nuviala et al. 2008; Sivadas y Baket-Prewitt, 2000; Papadimitriou y Karteroliotis, 2000; Murray y Howat, 2002; Alexandris \& Palialia, 1999; Afthinos et al. 2005 y Scudder, 2005), siendo estos aspectos básicos para el correcto funcionamiento y sostenibilidad de estos centros.

Por otro lado, existen también muchos estudios sobre el perfil del usuario del centro de fitness, pudiendo citar a Colado (1998), quien realizóun estudio en centros de Barcelona,Alicantey Valencia, obteniendo como resultado en la media de años de los usuarios, 31,25 años, bastante más baja esta edad que la de la población objeto de este estudio. Agustín (1995), también realiza otro estudio, argumentando que el usuario predominante es el joven de clase media-alta y con edades comprendidas entre los 18 y los 39 años, datos muy parecidos a los ofrecidos por

Fecha recepción: 15-01-10 - Fecha envío revisores: 15-01-10 - Fecha de aceptación: 15-01-10 Correspondencia: Jerónimo García Fernández

Avd. Ciudad Jardín, 20-22

41005, Sevilla

E-mail: jeronimo@us.es
Pinillos (2004), en el que el 35\% de los usuarios eran de edades comprendidas entre los 25 y 34 años, este mismo estudio, pudo comprobar que los mayores de 50 años, tan solo tenían un peso poco superior del $10 \%$. Otro resultado parecido lo encontramos en el realizado por Nuviala et al. (2008), en el que la edad media era incluso inferior a lo antes expuesto, 28 años.

Refiriéndonos al estudio de hábitos deportivos de los españoles (García Ferrando, 2006), existe un cambio de práctica deportiva ya que en la población de 15 a 74 años, se ha aumentado en 2 puntos la práctica de un deporte pero ha disminuido en 2 puntos, los que practicaban varios deportes. A su vez, parece ser que el perfil del que práctica más deporte es el del sujeto varón, de 15 a 34 años, con estudios superiores y con posición social alta, siendo el que menos practica, las mujeres de más de 65 años, sin estudios y con una posición social baja. Asu vez, los mayores de 55 años, tienen frecuencias de práctica semanal superiores al 50\% de los encuestados, y suelen hacer deporte sin competir (86\%).

La gimnasia de mantenimiento y el aeróbic, rítmica, expresión corporal, step o pilates, ocupan los primeros puestos de actividades más practicadas, teniendo porcentajes de práctica del 14\% y el 11,7\% respectivamente (gimnasia de mantenimiento en el primer caso, $\mathrm{y}$ las demás actividades en el segundo). Según este estudio, tan solo el 4,4\% de los mayores de 55 años, pertenece a un gimnasio o CFP, siendo los clubes privados y las asociaciones municipales, las que más asociados tienen (14,1\% y $12,5 \%$ respectivamente).

Así mismo, en el estudio realizado en Sevilla, sobre hábitos de los mayores en este municipio (Porras, 2007), se pudo comprobar que el deportemás practicado era la gimnasia demantenimiento con un 21,04\% de practicantes, la segunda más practicada la natación (16,50\%) y la tercera la carrera a pie (9,71\%). Así, según los distritos censales, en los distritos Este, Macarena Norte, Nervión, San Pablo / Santa Justa, Sur y Palmera / Bellavista, tienen los porcentajes más altos en gimnasia de mantenimiento (23,91\%, 44,44\%, 25,71\%, 27,59\%, 24,32\% y 55,56\%, respectivamente), en Macarena y Triana es la natación, la más practicada (16,98\% y 44\%, respectivamente, y en el Casco Antiguo y Cerro / Amate, los deportes de equipo (33,33\% y 15\%, respectivamente). Este estudio, también pone de manifiesto, las formas de práctica según distrito censal (gráfico 1).

Otros estudios se han centrado en la cuota de pago de los centros deportivos, como variable de fidelización (Triadó y Aparicio, 2004), obteniendo como resultados que el tipo de cuota familiar, es la estrategia 
que puede tener cierta independencia de la competencia, y que las cuotas de mayores, familiares e infantil forman un grupo diferenciado de las individuales, siendo los del primer grupo, miembros más fieles que los del segundo.

Hasta hace poco, no se había investigado mucho sobre gestión deportiva, y por lo tanto tampoco existían perfiles idóneos para estos puestos, teniendo cierta dificultad para encontrarlos (Martínez del Castillo, 2002, tesis), pero cada vez más, se empiezan a situar en estos puestos personas con mejor formación en la materia, que entienden y comprenden las necesidades de la sociedad actual. Así, para Gómez (2002), el perfil del gestor deportivo debería ser licenciado en CCAFYD / Magisterio de E.F. o TAFAD, vinculado al deporte, con capacidades de liderazgo, de entrega al trabajo y de trabajo en grupo, con fluidez de comunicación y debe ser una persona resolutiva.

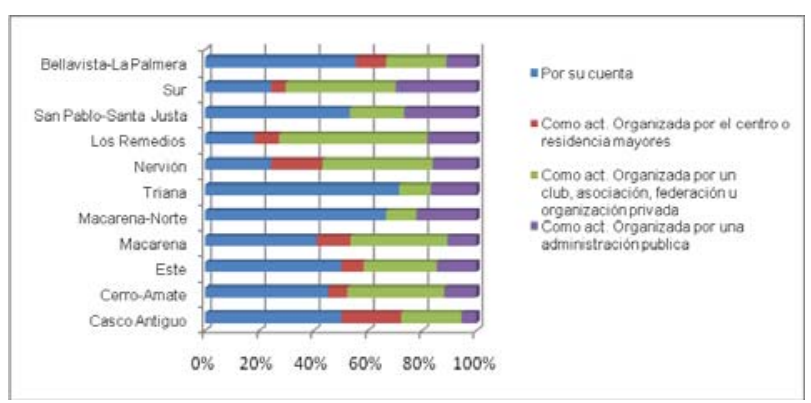

Figura 1: Formas de practicar deporte según distrito censal en el municipio de Sevilla Fuente: Porras, 2007, pág. 53.

Todo esto nos hace pensar, que el aumento de la creación de centros de fitness es hoy una realidad, y puede ser consecuencia del aumento de la práctica, y por la motivación de mejorar el estado físico y mental (Caracuel et al., 2003), pero las propuestas de programas y servicios de actividad física para mayores, están todavía distantes de la realidad existente, así según previsiones para la industria del fitness (The International Health, Racquet \& Sportsclub Association, 1990), se prevé que en 2010, hay más de 100 millones de usuarios (adscritos) en centros deportivos de todo el mundo, por lo que esto supone un aumento en necesidades y propuestas de mejoras para los clubes existentes y futuros.

El objetivo de este trabajo es el de comprobar la falta de ofertas existentes en CFP para personas mayores, así como el estudio de la inexistentepresencia de servicios específicos para este grupo de población en el municipio de Sevilla y su relación con los distritos censales.

\section{Material y método}

Para plantear nuevos servicios, potenciar la actividad física en personas mayores y abastecer la demanda evidente en centros privados, se debe primero saber la oferta existente en los CFP, por lo que se considera de interés lo planteado en esta comunicación.

En nuestro caso, el estudio se realizó en el municipio de Sevilla, y se utilizó como herramienta de recopilación de datos, el cuestionario. La muestra del presente estudio, lo forman 45 centros de fitness, de gestión privada, con diferentes extensiones en lo que a metros cuadrados se refiere, con diferentes números de socios, y en diferentes distritos censales del municipio de Sevilla. Asu vez, estos CFP, se dividieron por códigos censales, según aparece en la Web del ayuntamiento de Sevilla (http:// www.sevilla.org), siendo un total de 11 distritos los que conforman dicha división: Macarena, Macarena Norte, Nervión, Casco Antiguo, Este, Palmera / Bellavista, Sur, Cerro / Amate, Sur, Los Remedios y Triana.

Los datos se han comparado entre los propios centros, entre la oferta dentro del mismo distrito censal (tabla 1), y en relación con la población existente en cada distrito censal obtenidos del Instituto Nacional de Estadística (Porras, 2007).

\begin{tabular}{|l|c|c|}
\hline \multicolumn{3}{|c|}{$\begin{array}{c}\text { Tabla 1: Distribución de CFP por distritos censales y población mayor existente } \\
\text { Fuente: Elaboración propia a partir de Porras, 2007 }\end{array}$} \\
\hline DISTRRTO CENSAL & N DE CFP TESTEADOS & No POBLACIÓN MA YOR \\
\hline Casco a ntiguo & 6 & 17.465 \\
\hline Macarena & 4 & 26.366 \\
\hline Macarena Norte & 6 & 13.902 \\
\hline Nervión & 4 & 17.273 \\
\hline Cerro / Amate & 3 & 23.961 \\
\hline San Pablo / Santa Justa & 1 & 20.677 \\
\hline Triana & 5 & 17.911 \\
\hline Los Remedios & 2 & 9.200 \\
\hline Palmera / Bellavista & 3 & 8.602 \\
\hline Sur & 4 & 21.069 \\
\hline Este & 7 & 16.206 \\
\hline
\end{tabular}

Comentar también, que las actividades o programas que se plantean en algunos CFP como Pilates, Yoga o Taichi, no se han tenido en cuenta como actividades específicas, y sí las denominadas como «edad de oro», «mantenimiento» 0 «gimnasia de mantenimiento».

La hipótesis que planteamos, es que la oferta de cuotas específicas para personas mayores de 60 años, es muy escasa en relación a los CFP testeados, siendo también muy baja la oferta de programas o servicios específicos (actividades dirigidas específicas para mayores), con porcentajes bajos de cuotas específicas en los distritos censales con mayores números de población mayor.

\section{Resultados}

Se confirmó que la oferta de cuotas para personas mayores era casi inexistente en los CFP testeados, tan solo un 24\% (gráfico 2), siendo también muy baja la oferta de actividades específicas (20\%), como gimnasia demantenimiento para mayores en los mismos centros (gráfico $3)$.

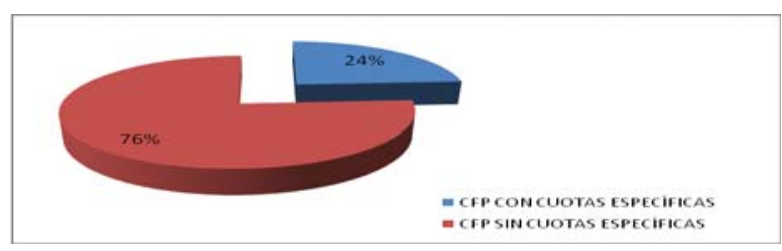

Figura 2: Oferta de cuotas específicas para mayores Fuente: Elaboración propia

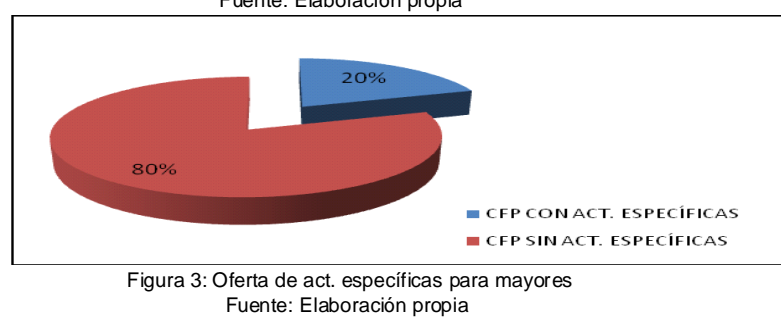

También observamos (gráfico 4) que en los distritos San Pablo / Santa Justa, Sur, Cerro / Amate, Palmera / Bellavista y Este, son los que tienen más CFP con cuotas específicas (100\%, 50\%, 33\%, 33\% y 29\%, respectivamente), así como los distritos que tienen menos cuotas específicas en sus CFP son los de Los Remedios, Macarena y Casco Antiguo (100\%,100\% y 83\%, respectivamente), siendo el precio medio de este tipo de cuota $34,62 \cdot$, teniendo la cuota más cara el distrito Sur con $41 \bullet$, y la más barata el Este con $25 \cdot$

Así mismo, los CFP no suelen tener programas ni actividades dirigidas específicas para las personas mayores, tan solo en el distrito censal San Pablo / Santa Justa, encontramos su existencia (100\%), y muy tímidamente en los distritos Macarena Norte (33\%), Este (29\%), y con un 25\% los distritos Macarena, Sur y Nervión. Los distritos Casco Antiguo, Cerro / Amate, Triana, Los Remedios y Palmera / 
Bellavista, muestran porcentajes inferiores al 25\% en cuanto a la disponibilidad de actividades específicas para mayores (gráfico 5).

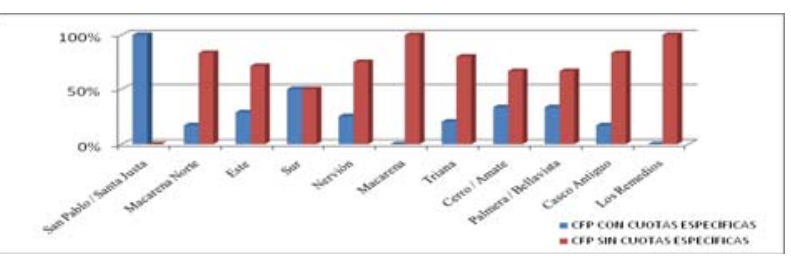

Figura 4: CFP con/sin cuotas específicas por distritos censales. Fuente: Elaboración propia

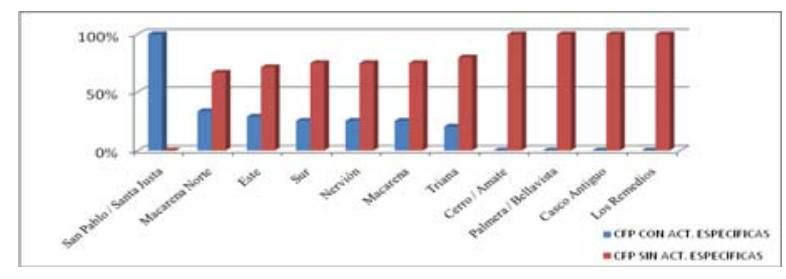

Figura 5: CFP con/sin actividades específicas por distritos censales Fuente: Elaboración propia

En referencia a la comparación de ofertas de cuotas y servicios específicos según número de población, el distrito Macarena teniendo el mayor número de mayores, tiene la décima posición en lo que se refiere a cuotas específicas, y la sexta posición en oferta de actividades específicas. Sin embargo el distrito Palmera / Bellavista, que es el que tiene menor número de mayores, ocupa el cuarto lugar en la oferta de cuotas específicas y el décimo lugar en la oferta de actividades específicas (tabla 2).

\begin{tabular}{|c|c|c|c|c|}
\hline \multicolumn{5}{|c|}{ Tabla 2: Posición de los distritos en relación a cuotas y act. espećficas } \\
\hline Distrito & Mayores & $\begin{array}{c}\text { Posición / } \mathbf{N}^{\mathbf{0}} \\
\text { habitantes }\end{array}$ & $\begin{array}{c}\text { Posición / C. } \\
\text { específicas }\end{array}$ & $\begin{array}{c}\text { Posición / Act. } \\
\text { específicas }\end{array}$ \\
\hline Macarena & 26.366 & 1 & 10 & 6 \\
\hline Cerro / Amate & 23.961 & 2 & 3 & 8 \\
\hline Sur & 21.069 & 3 & 2 & 4 \\
\hline San Pablo / Santa Justa & 20.677 & 4 & 1 & 1 \\
\hline Triana & 17.911 & 5 & 7 & 7 \\
\hline Casco Antiguo & 17.465 & 6 & 9 & 10 \\
\hline Nervión & 17.273 & 7 & 6 & 5 \\
\hline Este & 16.206 & 8 & 5 & 3 \\
\hline Macarena Norte & 13.902 & 9 & 8 & 2 \\
\hline Los Remedios & 9.200 & 10 & 11 & 11 \\
\hline Palmera / Bellavista & 8.602 & 11 & 4 & 9 \\
\hline
\end{tabular}

\section{Discusión}

Los CFP como centros de ocio, esparcimiento y lugar donde mejorar la condición física de sus usuarios, se prevé que es un lugar idóneo para la mejora física y psíquica de la población mayor. Así, es necesario que los gerentes y responsables de la gestión de los mismos, se encarguen de propiciar y ofertar servicios específicos, con materiales e infraestructuras adecuadas para este grupo de población.

Aún así, tan solo encontramos en los CFP testeados del municipio de Sevilla, un $24 \%$ de estas instalaciones con cuotas de abono específicas y un $20 \%$ de los mismos con actividades específicas, tales como «edad de oro» o «gimnasia de mantenimiento», programadas para tal público.

Así mismo, los distritos censales que más ofrecen las cuotas específicas son San Pablo / Santa Justa (100\%), Sur (50\%), Cerro / Amate (33\%) y Palmera / Bellavista (33\%), siendo los que menos las ofrecen Macarena (0\%), Los Remedios (0\%) y Casco Antiguo (17\%).

Por otro lado, el distrito San Pablo / Santa Justa (100\%), Macarena Norte (33\%) y Este (29\%), son los que ofrecen más actividades específicas.

Finalmente comentar que Macarena y Cerro / Amate, ocupando la primera y segunda posición en cuanto a número de población mayor, ocupan la décima y tercera posición en cuanto a oferta de cuotas se refiere, y sexta y octava posición en cuanto a actividades específicas ofertadas.

\section{Conclusiones}

A partir de los resultados obtenidos y centrándonos en la gestión de las cuotas y planificación de servicios / programas específicos para mayores, las principales conclusiones son las siguientes:

o El municipio de Sevilla cuenta con poca oferta de cuotas de abono específicas para personas mayores, en CFP.

o El municipio de Sevilla cuenta con poca oferta de actividades específicas para personas mayores, por parte de los CFP.

o De los distritos censales de Sevilla, los que mayor número de habitantes mayores tienen, son los que menos oferta de cuotas y servicios específicos disponen.

Para futuras investigaciones, sería interesante que se testeara un número mayor de CFP, ya que en este estudio no se ha hecho con la totalidad de los centros existentes, incluso es determinante el conocimiento sobre la percepción de este grupo de población, por parte de los gerentes y responsables de este tipo de instalaciones.

\section{Bibliografía}

Afthinos, Y., Theodorakis, N. D. y Nassis, P. (2005) Customers' expectations of service in Greek fitness centers. Managing Service Quality, 15 (3), 245-258.

Agustin, J. P. (1995). Sport Géographie et Aménagement. París: Nathan Université.

Alexandris, K. y Palialia, E. (1999) Measuring customer satisfaction in fitness centres in Greece: An exploratory study. Managing Leisure 4, 218-228.

Caracuel, J., Arbinaga, F. y Montero, J. A. (2003). Perfil socio-deportivo de dos tipos de usuarios de gimnasio: un estudio piloto. Revista de Psicología y Psicopedagogía, 2 (2), 273-308.

Colado Sánchez, J. C. (1998). Fitness en las salas de musculación. Barcelona: Inde. $2^{\mathrm{a}}$ edición.

García Ferrando, M. (2006). Posmodernidad y Deporte: Entre la individualización y la masificación. Madrid: CSD.

Gómez, A. M. (2002). El rol del Gestor Deportivo en los Municipios de la Comunidad Valenciana. Pasado, Presente y Futuro. Trabajo presentado en el II Congreso de Ciencias del Deporte, INEF, Marzo, Madrid.

IHRSA, 1990. www.cms.ihrsa.org

Instituto Nacional de Estadística (2007). www.ine.es

Martínez del Castillo, J. (2002). Bases para una planificación estratégica en la formación de los RRHH en gestión y dirección técnica deportiva. Trabajo presentado en el II Congreso de Ciencias del Deporte, INEF, Marzo, Madrid.

Murray, D. y Howat, G. (2002) The relationships among service quality, value, satisfaction, and future intentions of customers at an Australian sports and leisure centre. Sport Management Review, 5, 25-43.

Nuviala, A., Tamayo, J. A., Iranzo, J. y Falcón, D. (2008). Creación, diseño, validación y puesta en práctica de un instrumento de medición de la satisfacción de usuarios de organizaciones que prestan servicios deportivos. Retos. Nuevas Tendencias en Educación Física, Deporte y Recreación, 14, 10-16.

Papadimitriou, D. A. y Karteroliotis, K. (2000). The service quality expectations in prívate sport and fitness centres: A re-examination of factor structure. Sport Marketing Quartely, 9 (3), 15-27.

Pinillos, J. (2004). Winning the retention battle: Librando la batalla por la retención. Investigación y Marketing, 83, 39-42.

Porras, M. J. (2007). Hábitos y Actitudes de los Sevillanos Mayores ante el Deporte. Observatorio del Deporte de Sevilla. Sevilla: IMD.

Scudder, M. (2005, Mayo). Retention: Necessary But No Longer Enough. Fitness Business Pro, 21 (5), 46-46. Obtenido el 4 de Septiembre de 2008 de la base de datos Business Source Premier.

Sivadas, E., y Baket-Prewitt, J. L. (2000). An examination of the relationship between service quality, customer satisfaction, and store loyalty. International Journal of Retail \& Distribution Management, 28, 73-82.

Triadó, X. y Aparicio, P. (2004). El tipo de cuota como variable de fidelización de usuarios en Entidades Deportivas: Un reto actual de consecuencias futuras. Investigación y Marketing, 83, 31-38. 